\title{
Neue Persönlichkeiten für eine neue Gesellschaft. Zukunftsaneignung durch Selbsterziehung in der katholischen Quickbornbewegung während der Weimarer Republik ${ }^{1}$
}

\author{
Alexander MAIER
}

\author{
ARTICLE INFO \\ Article history: \\ Received 20 February \\ 2019 \\ Accepted 6 April 2020 \\ Available online 31 July \\ 2020 \\ Keywords: \\ Romano Guardini, \\ Quickborn youth \\ movement, self- \\ -education, Catholicism, \\ Weimar Republic, \\ kulturkritik, 'new man', \\ leader, contingency

\begin{tabular}{l}
\hline A. Maier \\
Saarland University $\bullet$ \\
Faculty of Philosophy $\bullet$ \\
66123 Saarbrücken \\
Germany $\bullet$ \\
amaier@mx.uni- \\
saarland.de
\end{tabular}

\begin{abstract}
New Personalities for a New Society. Appropriation of the

Future through Self-education in the Catholic Quickborn Movement during the Weimar Republic

The idea of 'new man' was a big topic in the 'Kulturkritik'-discourse as well as in educational discussions after World War I in Germany. Also the well-educated catholic milieu was engaged in this field. It was a time of great expectations and the protagonists hoped to build a new and better world - especially in the morals department. The precondition of this project was the education of the 'new man'. This article aims to analyse the engagement of the catholic Quickborn youth movement during the 1920 s to create this new personality, that was characterized as a leader figure for the youths in the movement itself but in the end also for the whole nation. Important questions are: How did the Quickborn youth movement pick up and modify the existing expectations of a new era for their special context or create their one views about it? How did they appropriate these views of future by self-education? And finally: Why did they operate in this way?
\end{abstract}

\section{Einleitung und Fragestellung}

Noch ganz unter dem positiven Eindruck, den die zweite Bundestagung der katholischen Jugendbewegung Quickborn auf der bei Würzburg gelegenen Burg Rothenfels offenbar auf ihn gemacht hatte, schrieb der Theologe Romano Guardini (1885-1968) kurze Zeit später in einem Brief an einen befreundeten Benediktiner die enthusiastischen Worte: „Zuerst war ich auf Rothenfels. [...] All mein Optimismus ist da droben gerechtfertigt worden. Die Menschen, die eine neue Welt bauen, sind da.“2 (Maier, 2017, S. 169). Damit brachte Guardini, der nur kurz nach diesem ersten Direktkontakt mit dem Quickborn zu dessen geistlich-geistigem Mentor avancierte, eine Zuversicht zum Ausdruck, die angesichts des für Deutschland negativen Kriegsausgangs, der Novemberrevolution von 1918 und dem damit

\footnotetext{
${ }^{1}$ This contribution is revised lecture which was presented in Prague, September 2018.

${ }^{2}$ Gemeint ist der Maria Laacher Benediktiner P. Kunibert Mohlberg.
} 
verbundenen politischen Systemwechsel sowie den sozio-ökonomischen und psychologischen Problemen der Nachkriegszeit verwundern mag. Gleichwohl stand Guardini damit nicht allein. Wie Rüdiger Graf hervorgehoben hat, war eine positive Sicht auf die Zukunft in der Weimarer Republik - insbesondere in der Berichterstattung und der Kommentierung des Zeitgeschehens in der Presse - vorherrschend (2008, S. 112). Graf diagnostiziert daher die Virulenz eines Wendebewusstseins: „Die Erwartung einer ,neuen Zeit' und eines ,neuen Deutschland' bzw. einer neuen Staats- und Wirtschaftsordnung [...] prägte die Erfahrung der Weimarer Republik von Beginn an. Das Gefühl, an einer Zeitenwende zu stehen, war weit verbreitet und konstituierte den Deutungshorizont gegenwärtiger Entwicklungen.“ (S. 143). In ähnlicher Weise hat Thomas Mergel insbesondere dem politischen Handeln jener Jahre die nicht unproblematische Orientierung an einer ,Erwartungsstruktur ' bescheinigt (2005). Optimismus und Erwartung verbanden sich mit der Vorstellung, die Gesellschaft auch tatsächlich verändern zu können, sofern man nur Sittlichkeit, Moral, Kultur oder religiöse Gesinnung der Menschen heben könne. Vor diesem Hintergrund gewannen sowohl die Eugenik als auch pädagogische Reformprogramme an Bedeutung. Diesbezüglich konstatiert Graf: „Da man annahm, neue Menschen könnten eine Veränderung der Gesellschaft und der Welt bewirken, ging es in der Eugenik durchaus um eine, Wende aller Weltalter', und in der Pädagogik herrschte bisweilen eine ,epochale Denkweise‘, um ,den neuen Menschen für die neue Zeit [...] zu bereiten'. Beide Diskurse sind signifikant für die Gestaltungseuphorie der Zeit, weil sie - stärker als das bei früheren Utopien der Fall war - den Menschen selbst zur Verfügungsmasse machten.“(2008, S. 194).

Die Zeitenwende-Rhetorik wurde auch im religiösen Feld aufgegriffen, wobei insbesondere der Katholizismus nach dem Zusammenbruch des Kaiserreichs und des mit ihm untrennbar verknüpften „furor protestanticus“3 (Gerber, 2012, S. 382) - so eine zeitgenössische Einschätzung - zu einer durchaus selbstbewussten Rolle in der Weimarer Republik fand, deren deutlichste Zuspitzung sicherlich im ,Siegkatholizismus' eines Erich Przywara (18891972) lag, der die katholische Kirche als alleiniges Heilmittel gegen die Krisenerfahrungen der Nachkriegsjahre und die Verunsicherungen der modernen Gesellschaft beschwor (Ruster, 1994, S. 76).

Die Quickbornbewegung, die im Folgenden nun im Fokus stehen soll, geht auf die Initiative des lebensreformerisch wie reformtheologisch geprägten Priesters und Pädagogen Bernhard Strehler (1872-1945) zurück, der seine Schüler am Bischöflichen Konvikt in Neiße 1908/09 zur Gründung eines Abstinentenzirkels angeregt hatte. Dieser konnte sich relativ schnell an vielen Schulen im Kaiserreich etablieren und bildete im Kontext der Entwicklung einer nicht am Alkohol orientierten Geselligkeit jugendbewegte Formen aus, die, so Strehler, die Jungen und Mädchen des Quickborn in einem ,Jugendreich` zusammenführen sollten. Damit verbanden sich im Quickborn von Anfang an das reformpädagogische und das religiöse Feld. Die Suche nach der Balance beider Bereiche blieb ein Charakteristikum der Bewegung. In den 1920er Jahren beeinflusste der Quickborn auch die deutschsprachige katholische Jugend in der Tschechoslowakei, so insbesondere den 1920 gegründeten Bildungs- und

\footnotetext{
${ }^{3}$ So formulierte es 1918 der Kapuzinerpater Johannes Chrysostomus Schulte.
} 
Wanderbund Staffelstein, in den sich die sudetendeutschen Quickborngruppen in der Folge auch integrierten (Langhans, 1990, S. 65-67; Kasper, 2016, S. 42-48).

Hier soll nun analysiert werden, wie sich nach 1918 die gesellschaftlich präsente Zeitenwende-Rhetorik im Quickborn niederschlug und wie Zukunft hier formatiert und angeeignet wurde. Dazu dienen folgende Leitfragen: Inwiefern wurden in der Bewegung reformpädagogisch-jugendbewegte Motive aufgegriffen? Wie modifizierte sie diese im Hinblick auf ihren religiös-katholischen Kontext? Wie sah das Idealbild der neuen Persönlichkeit und der neuen Gesellschaft aus? Wie bzw. wodurch sollte die Selbsterziehung der Quickbornerinnen und Quickborner angeregt werden?

\section{Zukunftsbilder und Zeitenwende-Rhetorik}

Genauso wie die Publikationsorgane der Freideutschen ${ }^{4}$ (Fiedler, 1989, S. 160), hatten auch die Veröffentlichungen des Quickborn das Ende des Ersten Weltkrieges kaum thematisiert und wenn doch, dann wurden die Kriegserfahrungen spiritualisiert, rationalisiert oder pädagogisiert. Immerhin stellte ein Autor im ,Quickborn', der Zeitschrift der Bewegung, fest, dass durch das Kriegsende neue Herausforderungen entstanden seien. Diese wurden jedoch nicht näher ausgeführt. Auffallend ist der optimistische Grundzug des Beitrags, der einerseits betont, dass die „Jugendseele“ (Wahreck, 1918/19, S. 148) von den Ereignissen unberührt geblieben sei, so dass gerade auch der Quickborn sein Wirken ungebrochen fortsetzen könne. Andererseits drückt der Verfasser seine Zuversicht aus, dass die Einheit des deutschen Volkes als sozialer Körper nicht in Gefahr wäre, wofür gerade auch das Engagement der Jugend Sorge trage (S. 148).

Spätestens mit dem zweiten Quickborntag, der vom 7. bis 10. August 1920 auf der ein Jahr zuvor erworbenen Burg Rothenfels mit rund 1500 Teilnehmerinnen und Teilnehmern stattfand, rückte die Diskussion um die Zukunft der Gesellschaft immer deutlicher in den Vordergrund, wenngleich es im Zusammenhang mit dem Einsatz für die Abstinenz auch schon vor und während des Weltkrieges gesellschaftsreformerische Bestrebungen gegeben

\footnotetext{
${ }^{4}$ In der Forschung zur Jugendbewegung wird häufig zwischen den freien bzw. freideutschen und den ,ideologisch ' gebundenen Gruppen - die sich Kirchen oder politischen Richtungen verpflichtet gefühlt haben unterschieden. Insbesondere Jürgen Reulecke hat diese Unterscheidung stark gemacht. Für ihn stellt das Bekenntnis zur jugendlichen Autonomie das entscheidende Kriterium für die Zugehörigkeit zur freideutschen Jugendbewegung dar. Die konfessionellen Gruppen hätten ihrerseits lediglich viele Formen von den Freideutschen übernommen (Reulecke, 2013, S. 54). Zwischenzeitlich wird diese Forschungsmeinung zu Recht stark hinterfragt, denn auch die freideutsche Jugendbewegung bzw. der Wandervogel kam nicht ohne gewisse Rückbindungen - etwa an die von der Lebensreform beeinflussten Eltern oder Lehrer - aus (Herrmann, 2006, S. 55-56). Zudem waren die Bünde, die sich 1913 auf dem Hohen Meißner getroffen hatten um dort ihr Bekenntnis zur Autonomie der Jugend zu verabschieden, überwiegen völkisch und antisemitisch orientiert. D.h., sie waren keineswegs frei von den bestimmenden Ideologien jener Zeit. Im Übrigen wurde die Freiheitsformel vom Hohen Meißner schon bald von Pädagogen kritisiert und durch die Marburger Formel (1914) deutlich relativiert (Niemeyer, 2013, S. 71-75, 165 u. 181-183). Für die Zugehörigkeit des Quickborn zur deutschen Jugendbewegung sprechen eine Reihe von Fakten. Zu nennen wären etwa die Selbstwahrnehmung der Quickbornjugend (Kaltenhäuser, 1919/20, S. 104-106) aber auch die Fremdwahrnehmung (z.B. durch den damals bedeutenden Pädagogen Friedrich Wilhelm Foerster, 1869-1966; Foerster, 1923, S. 255). Auch die an sich problematische Quellen-Dokumentation zur deutschen Jugendbewegung von Werner Kindt zählt den Quickborn selbstverständlich dazu (Kindt, 1963).
} 
hatte (Maier, 2018a). Dies hing sicherlich auch mit der Rolle der Älteren im Quickborn zusammen, die bei dieser Tagung auch die Gründung einer eigenen Zeitschrift beschlossen hatten, die stärker ihre Bedürfnisse als Studierende oder sogar schon im Beruf stehende Erwachsene berücksichtigen sollte. Das neue Organ ,Die Schildgenossen“, sollte daher stärker eine Kulturzeitschrift, als ein Mitteilungsblatt des Bundes sein. Die Gründung der Zeitschrift markiert zugleich die sich herausbildende Konkurrenz zweier Vorstellungen des Quickborn: Jugendbewegung oder Kulturbewegung (Marmetschke, 2006, S. 293-295).

Die erste Ausgabe der neuen Zeitschrift, Die Schildgenossen“ vom Oktober 1920 dokumentiert die inhaltlichen Diskussionen des zweiten Quickborntages. Es ging dem Quickborn dort um nichts Geringeres, als die Erneuerung des menschlichen Lebens (Liedl, 1920, S. 13) im Allgemeinen und die Wiederbelebung des christlichen Charakters des deutschen Volkes und seine sittliche Reform im Besonderen (Steidle, 1920, S. 18-19). Wenn bisweilen die Rhetorik die nationale Perspektive auf der Basis eines globalen Katholizismus überschritt, indem sogar das Ziel der „Welterneuerung“ (Hörle, 1921, S. 22) ins Auge gefasst wurde, steigerte sich jugendbewegte Zuversicht ins Unendliche. Besonders greifbar werden diese Ziele in einem Kommentar des katholischen Intellektuellen Hermann Platz (1880-1945) $\mathrm{zu}$ Geschehen und Atmosphäre des Quickborntages. Dieser konstatierte zunächst kulturkritisch: „Der Krieg und die Ereignisse der Nachkriegszeit haben die Eiterbeule, die in der Großstadt gereift ist, aufbrechen lassen. Das Geld gewinnt im heutigen Schieber-, Wucher- und Protzentum die letzte Bedeutung, die ihm als Symbol der vergangenen Kulturentartung zukommt." Daraufhin beschrieb Platz die Zukunftsvision, die auf der Versammlung spürbar gewesen sei und gibt sich zugleich darin sicher, dass die Wende zu einer besseren Zeit nicht nur ersehnt, sondern in einer neuen Haltung und einem neuen Miteinander auch schon spürbar gewesen sei. „Die schicksalshafte Verflochtenheit“ mit den von ihm kritisierten Zuständen, so Platz, ,ist unser tiefes Leid und das Ziel unserer heimlichen Sehnsucht ist es, irgendwo an das andere Ufer zu gelangen, wo das neue Seelentum zu sprießen beginnt. Das war mein Erlebnis auf Burg Rothenfels: Diese Abgelegenheit und Erhabenheit waren Leben und Sinn. Da waren junge Menschen, die diese Wirklichkeit brauchten, um ihrem Sein Genüge zu tun. Hier riß der Faden des Alltags ab, hier zerbrach die Allmacht des Geldes, so weit weg, so hoch drüber war es. Hier war Seele an Seele gelehnt, Wille an Wille gereiht und gerichtet, und der Flügelschlag des neuen Geistes war vernehmbar. (...) Die Aufgeschlossenheit und Bereitschaft der Seelen beweisen, daß die Zeit vor einer Wende steht“" (1920, S. 30).

\section{Die neue Persönlichkeit: Der Führer/die Führerin}

Im Beitrag von Platz klingt zugleich die Vision einer neuen Persönlichkeit an. Denn nur dieser neue Mensch wird in der Lage sein, diese Wende aktiv zu vollziehen und die bevorstehende Zukunft zu ergreifen: „Hier bereiten sich ganze Menschen zu ganzen Taten. Die Welt hat genug der Halbheit. Wie lachte mein Herz, daß hier nicht mehr in Formeln gedacht und auf Kommando gehandelt wird. Die Welt hat genug der Puppen (...). Es ist eine Sehnsucht und eine Richtung deutlich gemacht durch Worte, die aus echter Begeisterung 
kommen“ (ebd.). Interessant ist, dass diese neue Persönlichkeit - Platz spricht vom „intensiven, herrischen, monumentalen“ (1920, S. 31) Persönlichkeitstyp -, die im Laboratorium des Quickborn entstehen soll, einerseits als selbstbestimmter Charakter gedacht wird, andererseits sollen diese neuen Menschen durch ein gemeinsames, vom „Flügelschlag des neuen Geistes“ (1920, S. 30) gewecktes Ziel zu einer Gemeinschaft verbunden sein. Insofern trifft auch für den konfessionellen Quickborn die Analyse Peter Schröders zu, dass die Jugendbewegung ,das Ergriffensein junger Menschen von sich selbst (ist), das sich in der Bildungsidee vom neuen deutschen Menschen manifestiert und in der autonomen Institution bündischer Gemeinschaft gelebt wird“ (1996, S. 44-45.). Letztlich ist die hier modellierte neue Persönlichkeit eine Führergestalt, die, so Jürgen Reulecke, für die Jugendbewegung in der Weimarer Republik charakteristisch war (2013, S. 52-57). In den Debatten um das Führerverständnis im Quickborn kristallisierten sich vor allem die Bereitschaft zum Dienen sowie die Tugend der Demut als zentrale Merkmale heraus (Ponschab, 1922, S. 191-192). Exemplarisch dafür steht etwa die Beschreibung der idealen Führerpersönlichkeit von Georg Mahlich von 1921: „In Wahrheit Führer kann daher nur sein der Demütige, der ganz Einfache, der prunkende Worte scheut, der Gütige, der Böses mit Gutem vergilt, der nie verletzt [...], der gibt und empfängt und innen voll Liebe ist, der stets Wachsame, der im Bewußtsein der eignen Schwäche unaufhörlich ringt. - In Wahrheit Führer ist einer nur dann, wenn er nicht mehr sein will als der Bruder aller, wenn er die guten Menschen sucht und sie gern in selbstloser Bewunderung sich zum Vorbild nimmt nur dann, wenn er es selbst nicht weiß, wie sehr und wann er anderen Vorbild ist.“(1921, S. 146). Wie unschwer zu erkennen ist, orientiert sich das Führerverständnis am Vorbild bzw. der Nachfolge Jesu. Insofern der Einzelne dieses Bild internalisiert und „der ganze Kerl ein ,imitator Christi““ (Spörl, 1923, S. 258) wird, gewinnt die neue Persönlichkeit Kontur. Auch wenn aufgrund der religiösen Rückbindung des Quickborn das Ideal des Führers nicht das des Herrschers aus eigenem Recht sein konnte - einen Typus des Führers, den der spätere austrofaschistische Volksbildner Viktor Winkler-Hermaden (1895-1942) in seiner psychologischen Studie über den Jugendführer von 1927 insbesondere der Jugendbewegung zugeordnet hatte (Janke, 2010, S. 349), so ist der hier konturierte christliche Führer doch keinesfalls ein Schwächling (1927, S. 43). Vielmehr gewinnt seine Führerpersönlichkeit Bedeutung und Kraft durch seine Bezugnahme auf Jesus Christus. In seiner Berufung auf die Objektivität des christlichen Glaubens überbietet der christliche Jugendführer sogar den freideutschen Führertypus, der sich letztlich nur auf seine Subjektivität zu gründen vermag, wenngleich es natürlich auch hier Vorstellungen von evident verstandenen Rückbindungen gab - so etwa das Führercharisma oder die Kampferfahrung im Krieg, die den Wandervogelsoldaten einen quasi mystischen Zugang zum Verständnis des Volkes ermöglicht hätte (Reulecke, 2001, S. 44 u. 81; 2011, S. 162). Damit wird der Quickbornführer auch noch zum „Wegweiser mit Rat und Tat, mit Wort und Beispiel“ (Böer, 1922, S. 4) für die freideutsche Jugend. Dies zeigt, dass die Akteurinnen und Akteure im Quickborn den von freideutscher Seite erhobenen Vorwurf, ihre Unterordnung unter die Autorität (z.B. die der Kirche), wäre „,ein Aufruf zum schwächlichen, verantwortungslosen Leichtsinn“ und 
keinesfalls ein „Zeichen von Kraft“ (Kelber, 1921, S. 114) und Jugendbewegung und Katholizismus würden sich nicht miteinander vertragen (S. 115), einfach zu ihrem Qualitätsmerkmal stilisierten, in dem sie in Gehorsam und Hingabe eine höhere Form von Freiheit sahen. Guardini sprach hier vom ,schöpferischen Gehorsam‘ (Maier, 2017, S. 176). Dieser, so Guardini, ,gibt [...] aus der Fülle heraus; er tut mehr als er muß; er schenkt großmütigen Gehorsam und macht ihn dadurch zur eigenen Schöpfung“"(1920, S. 38), so dass gerade dadurch die freie und starke Persönlichkeit hervorgebracht wird. Die Abgrenzung zu den Freideutschen geht also mit einer Steigerung der eigenen Bedeutung einher, so dass man sagen könnte, der Quickborn verstand sich gerade aufgrund seiner Rückbindung an Autoritäten als die eigentliche oder bessere Jugendbewegung. ${ }^{5}$ Die Bedeutung der Bindung an Autorität statt einer grenzenlosen Freiheit, traf offensichtlich auch die Stimmung der Quickbornjugend und kann daher nicht nur als eine auf Guardini begrenzte Position reduziert werden. Exemplarisch soll auf einen Brief der Quickbornerin Antonie hingewiesen werden. Darin beschreibt sie, dass sie während ihrer Zeit im Wandervogel immer auf der Suche nach der Wahrheit gewesen wäre. Diese habe sie dort jedoch nicht finden können. Daher ist sie froh, mittlerweile zum Quickborn gestoßen zu sein. Sie hält fest: „Ich habe geprüft und bin Quickbornerin geworden. Jetzt besitze ich Christus als Gott, als Führer, als Erlöser. [...] Ich bin froh, daß ich als Quickbornerin einen sicheren Weg habe.“ (Antonie, 1921, S. 107). Dies zeigt die offensichtlich entlastende Funktion einer dezidierten religiösen Bindung.

Dass das Ideal der Nachfolge Christi ganz offenbar mit dem Bild des neuen katholischen Menschen im Quickborn vermittelbar war, mag aus heutiger Sicht erstaunen und entbehrt auch nicht einer theologischen Problematik, die jedoch für die damaligen Akteure offenbar so nicht bestanden hat. Vielmehr war gerade die Hoffnung auf eine neue, bessere Zukunft wie der Weg dazu religiös anschlussfähig, mehr noch: Die Rückgewinnung der Souveränität über die im Zuge der Moderne disparat und unverfügbar gewordene Welt schien nur noch religiös möglich (Makropoulos, 1994, S. 201). Da dieses antimoderne Projekt jedoch zugleich unter den Bedingungen der modernen Gesellschaft stattfand, war es auf die Kooperation mit innerweltlichen, auf Ganzheit und Objektivität zielenden Konzepten angewiesen (Ruddies, 1994, S. 26). In der praktischen Umsetzung z.B. des Führerideals und des damit korrespondierenden Gemeinschaftskonzepts fanden die Quickborner die Möglichkeit aus ihrer Sicht zugleich gesellschaftlich relevante und religiöse Erfahrungen zu machen, die ihnen die Einnahme eines Standpunktes jenseits der ambivalenten Welt ermöglichte. Dadurch konnten

\footnotetext{
${ }^{5}$ Über das Verhältnis von Freiheit und Bindung diskutierten wichtige Stichwortgeber aus dem freideutschen Lager mit Guardini. Zwei solcher Dialoge Guardinis - mit Wilhelm Kelber (1901-1967) und mit Max Bondy (1892-1951) - sind dokumentiert (Kelber 1921, S. 113-115; Guardini, 1921, S. 115-120; Guardini und Bondy, 1921/1963, S. 274-302). Eine Doppelmitgliedschaft in Quickborn und Wandervogel wurde durch den westdeutschen Quickborn-Vertretertag 1919 ausgeschlossen. Dies geschah offensichtlich aus religiösen Gründen aber auch, weil der Wandervogel seine ursprünglichen Ideale verraten habe. Der Bericht des Vertretertages hält fest: „Die freideutsche Jugend, anfangs interkonfessionell, ohne religiöse Bestrebungen, ist allmählich von ihrem Hochziel, dem Leben aus innerer Wahrhaftigkeit und eigener Verantwortung abgewichen. Ihre Weltanschauung ist ein Durcheinander von verblaßtem Christentum, Pantheismus und Materialismus.“ (Alumnus, 1919/20, S. 52). Punktuelle Kooperationen (Spiele, Feste) zwischen einzelnen Quickborn- und Wandervogelgruppen werden von manchen Stichwortgebern unter bestimmten Bedingungen befürwortet (Rocholl, 1922, S. 36-37).
} 
sie ihre erhofften Zukunftsentwürfe annähernd in religiöse Gewissheit verwandeln und somit auch einen katholischen Führungsanspruch erheben. Diese Entwicklung im Quickborn ist im Kontext eines Wandels katholischer Religiosität insgesamt zu sehen. Diese ließ immer mehr die feminin konnotierte Herz-Jesu-Spiritualität des 19. Jahrhunderts hinter sich und bildete zunehmend und besonders offenkundig in den 1920er Jahren, eine offensive ,Christkönigsfrömmigkeit‘ mit tendenziell maskulinem Beiklang sowie einem totalitären Führungsanspruch der Kirche aus (Weichlein, 2015, S. 95).

\section{Pädagogisierung der neuen Persönlichkeit}

Die Umsetzung des Führerbildes versuchte man v.a. durch Selbsterziehung zu erreichen. Die Organe des Quickborn boten in ihren Beiträgen dazu vielfältige Anregungen und veröffentlichten regelmäßig Literaturhinweise und Rezensionen, in der auf geeignete Literatur unterschiedlicher Genres aufmerksam gemacht wurde. Empfohlen wurde etwa Fachliteratur zur Abstinenz und zu Themen der Sozialreform (z.B. Koch, 1913; von Bunge, 1907) sowie Werke mit pädagogischem Inhalt, in denen z.B. die charakterstarke Persönlichkeit thematisiert wurde (z.B. Foerster, 1917; Klug, 1920). Daneben wurde auch auf Romane, wie z.B. auf das in der Jugendbewegung allgemein viel gelesene Kultbuch ,Helmut Harringa“ hingewiesen (Popert, 1910). Aber natürlich zählten auch Romane von dezidiert katholischen Schriftstellerinnen und Schriftstellern dazu. Zu nennen wäre etwa der Roman ,Ritas Briefe“ (Handel-Manzetti, 1915). Die Hinweise auf spirituelle Literatur (z.B. Br. Bardo, 1916) sowie auf Zeitschriften wie ,Das Heilige Feuer' dürfen nicht vergessen werden. Diese Zeitschrift, in der sowohl antisemitische als auch völkische Beiträge erschienen (Weiss, 2014, S. 90-91), empfahl Strehler insbesondere den älteren Quickbornerinnen und Quickbornern zur Lektüre, wie ein Hinweis deutlich macht: „Es ist seine Art (gemeint ist die Zeitschrift ,Das Heilige Feuer', A. M.), in die Tiefe $\mathrm{zu}$ gehen und in die Höhe $\mathrm{zu}$ streben. Glaubensinnige Frömmigkeit, Volkstum, Gemeinschaftsleben, Natur und Bildung werden von ihm mit viel Ernst und Eifer gepflegt. Für die oberen Klassen und besonders für Groß-Quickborner ein zuverlässiger Führer in die Aufgaben unserer Zeit. “6 (Strehler, 1919/20, S. 114). Daneben gab es in den Publikationsorganen des Quickborn auch negative Buchbesprechungen oder Warnungen vor bestimmter Literatur. So wurde etwa gegen Kriegsende vor den Büchern des Ullstein-Verlages gewarnt, denen u.a. moralische Laxheit vorgeworfen wurde. In einer solchen Warnung heißt es: „Heute sind den meisten Soldaten in der Heimat und in Feindesland, vielen Arbeitern und Arbeiterinnen, vielen Schülern und Schülerinnen diese Romane fast tägliche Geistesnahrung geworden. Und welcher Art sind sie? Eine schwüle Sinnlichkeit kennzeichnet die meisten von ihnen. Seichte sinnliche Anschauungen werden durch sie in breiteste Schichten unseres Volkes getragen. Das wird nun allmählich als eine große Gefahr erkannt. [...] Helft auch ihr diesen Schädling unseres Volkes bekämpfen, d.h. kauft nie ein Ullsteinbuch, auch wenn es einmal harmlos sein sollte, klärt andere über die

\footnotetext{
${ }^{6}$ Als Groß-Quickborner wurden seit 1920 die Älteren im Quickborn bezeichnet, die z.B. schon in das Berufsleben eingestiegen waren. Diese Altersgruppe bildete ab 1920 den Älterenbund Groß-Quickborn. Bereits 1924 wurden Jüngeren- und Älterenbund wiedervereinigt (Bundesleitung, 1924, S. 2).
} 
Ullsteingefahr auf und sucht sie für den Kampf gegen sie zu gewinnen!“7 (Eine Aufgabe für Quickborn, S. 133).

Die gelebte Abstinenz und die Werbung dafür, Sport, Spiel, Liturgie und Kommunionempfang sowie die Pflege einer persönlichen Beziehung zu Christus, der nun selbst als Führer zu individueller Ganzheit bezeichnet wurde, stellten weitere Elemente einer theologischen Quickborn-Pädagogik dar (Maier, 2018b). Während der Weimarer Jahre kam es - nicht zuletzt durch das Engagement Romano Guardinis - zu einer Differenzierung zwischen den Jüngeren und Älteren im Quickborn und damit auch zu teilweise unterschiedlichen Schwerpunktsetzungen im Erziehungsverständnis, die schließlich auch zu Konflikten und zur vorübergehenden Auflösung des Quickborn 1926 führte (Marmetschke, 2006, S. 292). Setzte sich bei den Jüngeren im Quickborn zunehmend der Gedanke der ,Jungenschaft' durch, der zu einer straffen, auf den Führer bezogenen Organisation und der Betonung von Fahrt und Spiel sowie teilweise einer Marginalisierung der Mädchen führte, sammelte Guardini die Älteren zu kulturellen Bildungsveranstaltungen auf Burg Rothenfels (Klönne, 1990, S. 142-143). Diese entwickelte sich in der Folge, so konstatiert Katja Marmetschke, ,immer stärker zu einem Ort intellektueller Auseinandersetzungen, zu einer unkonventionellen katholischen Bildungsakademie“ (2006, S. 292).

Im Quickborn geschah Selbsterziehung schließlich auch immer in Bezug zur Lehre der katholischen Kirche, die nicht nur, aber doch insbesondere von Guardini als die entscheidende Instanz der Lebensgestaltung eingespielt wurde. Insofern musste immer wieder versucht werden, zwischen der Freiheit der Jugend und der nicht hinterfragbaren Autorität der Kirche zu vermitteln. Dabei wurde z.B. zwischen wahrer und entarteter Jugend unterschieden, wobei der Wahrheitsanspruch durch die Katholizität der Quickbornjugend als garantiert betrachtet wurde, da Katholizismus per se „Verjüngung, ewige Jugend“ (Becker, 1922, S. 5) sei. Schließlich ist die Arbeit an sich selbst, die Innenarbeit zu nennen, denn nicht „Barett und Kluft“ machen den Einzelnen zum Quickborner, sondern, so Anton Thill, „der Kampf mit dem eigenen widerspenstigen Ich, der Wille, sich erst selber (...) zuerst einmal zu dem umzuformen, wie ich mir einen Quickborner denke [...]“(1922, S. 17).

\section{Resümee}

Nach 1918 haben junge Frauen und Männer und ihre Mentoren in der Quickbornbewegung im Spannungsfeld zwischen Jugendbewegung und religiöser Bindung versucht, Zukunftsperspektiven $\mathrm{zu}$ entwickeln, um auf die vielfältigen Herausforderungen der Gesellschaft in der Zwischenkriegszeit $\mathrm{zu}$ antworten. Damit wollten sie sich ihrer persönlichen Zukunft wie der Zukunft des deutschen Volkes ideell versichern, sich aktiv an der Gestaltung der neuen Gesellschaft - wenn auch nicht im Sinne liberaler Demokratie beteiligen und sich in beidem als selbstwirksam erleben, um darin letztlich Sinnsicherheit zu erfahren (Groppe, 2008, S. 75-81). Bildungsgeschichtlich interessant sind dabei v.a. zwei Aspekte. Das Beispiel des Quickborn zeigt erstens, dass pädagogische Reformprogramme,

\footnotetext{
${ }^{7}$ Der Beitrag bezieht sich vermutlich auf die Bücher der preisgünstigen Buchreihe ,Rote Ullsteinbücher‘. Ullstein, 1877 in Berlin als Zeitungsverlag gegründet, gab seit 1903 auch Bücher heraus (Oels, 2015).
} 
gerade auch entlang des Jugendmotivs, - entgegen der zumeist in der historischen Forschung dominanten Annahme - keineswegs nur ein Kind des Kulturprotestantismus gewesen sind, sondern Anfang des 20. Jahrhunderts auch im Katholizismus möglich waren. Damit verbunden und erstaunlich ist zweitens, wie stark der Katholizismus - und dies zeigt das Beispiel des Quickborn - in die Weimarer Gesellschaft involviert war. Möglich wurde dies gerade durch eine aus heutiger Sicht selbstverständlich ambivalente Kulturkritik, die als Motor der Kommunikation zwischen Glauben und säkularer Moderne fungierte (Gerber, 2012, S. 374 u. 388-390). Diese brachte, theologisch gesehen, bisweilen fundamentalistische Zuspitzungen hervor, wenn etwa behauptet wurde, „daß nur eine christliche Kultur uns retten kann“ (Liedl, 1920, S. 15). Pädagogisch redete sie vielfach einem problematischen Heroismus das Wort. Zugleich aber formten sich junge Menschen selbst nach idealen Werten in der Verknüpfung reformpädagogischer, jugendbewegter und konfessioneller Motive und gestalteten damit den Zukunftsdiskurs der Weimarer Gesellschaft aktiv mit. Die dabei entwickelten ideologischen oder politischen Optionen changierten zwischen christlichem Sozialismus und katholischem Konservatismus und schlossen auch völkisches Denken nicht aus (Richter, 2000, S. 191; Klönne 1981, S. 51-64; Maier, 2018b, S. 88-89). Das integrative Moment dieser nur auf den ersten Blick nicht miteinander vermittelbaren Positionen ist der die Weimarer Jahre entscheidend markierende Versuch, die moderne Erfahrung der Zersplitterung von Subjekt und Welt religiös wieder in den Griff zu bekommen (Makropoulos, 1994, S. 211). Mit seiner Kulturkritik wie seiner Zukunftsvision und ihrer Pädagogisierung in der neuen Persönlichkeit war der Quickborn daher eine katholische Variante eines für nach 1918 in Deutschland charakteristischen Reaktionsmusters auf eine gesellschaftliche Modernisierung bisher unbekannten Ausmaßes. Der Preis dieser Suche nach Sinnsicherheit, wie sie in der ,Hypostase“ des neuen Menschen zum Ausdruck gebracht wurde, war erstens ein Zugriff auf die Geheimnishaftigkeit Gottes und dessen eigentlich unverfügbaren Wirkens (Fuchs, 2005). Sie war zweitens das Bemühen um eine Integration individueller Erfahrung und Sinnsuche in die Ideologie des Quickborngeistes, wie sie für religiöse Organisationen charakteristisch ist (Taylor, 2013 S. 12). Im Verfügen wollen über diese Kontingenz des Geheimnisses Gottes wie über das individuelle Suchen und Fragen der Einzelnen lag letztlich die theologische wie pädagogische Brisanz des Quickbornprojekts. 


\section{Quellen- und Literaturverzeichnis}

\section{Quellen}

ALUMNUS, Josef, 1919/20. Zwei Tage in Hamm. Quickborn. 10. Jg., H. 3, S. 51-53.

ANTONIE, 1921. Brief zu den Unterschieden zwischen Quickborn und Wandervogel. Quickborn. 9. Jg., H. 4/5, S. 107-108.

BECKER, Willy, 1922. Jugend. Quickborn. 10. Jg., H. 1, S. 4-6.

BÖER, Ludwig, 1922. Sinn und Aufgabe unserer Zeit. Quickborn. 10. Jg., H. 1, S. 4.

BRUDER BARDO, 1916. Deutsche Gebete. Wie unsere Vorfahren Gott suchten. Freiburg i. B.: Herder.

BUNDESLEITUNG, 1924. An alle im Bund. Quickborn. 12. Jg., H. 1, S. 1-4.

BUNGE, Gustav, von, 1907. Die Alkoholfrage. Basel: Schriftstelle des Alkoholgegnerbundes. EINE AUFGABE FÜR QUICKBORN, 1918/19. Quickborn. 6. Jg., H. 9, S. 133.

FOERSTER, Friedrich Wilhelm, 1923. Jugendseele, Jugendbewegung, Jugendziel. Zürich München: Rotapfel.

FOERSTER, Friedrich Wilhelm, 1917. Jugendlehre. Ein Buch für Eltern, Lehrer und Geistliche. Berlin: Georg Reimer.

GUARDINI, Romano u. BONDY, Max, 1921/1963. Jugendbewegung und Katholizismus. Eine Aussprache zwischen Max Bondy und Romano Guardini. In: KINDT, Werner, Hrsg. Grundschriften der Deutschen Jugendbewegung. Düsseldorf - Köln: Diederichs, S. 274-302.

GUARDINI, Romano, 1921. Vom Sinne des Gehorchens. Erwiderung auf Wilhelm Kelbers Kritik meines Aufsatzes. Die Schildgenossen. 1. Jg., H. 4, S. 115-120.

GUARDINI, Romano, 1920. Vom Sinne des Gehorchens. Die Schildgenossen. 1. Jg., H. 2, S. 33-41.

HANDEL-MAZZETTI, Enrica, 1915. Ritas Briefe. Saarlouis: Hausen.

HÖRLE, Heinrich, 1921. Beitrag in der Rubrik ,Aus der Bewegung‘. Quickborn. 9. Jg., H. 1, S. 22.

KALTENHÄUSER, Margarete, 1919/20. Was ich von der Burg heimbringe. Quickborn. 7. Jg., H. 7, S. 104-106.

KELBER, Wilhelm, 1921. Vom Sinne des Gehorchens. Kritik des gleichnamigen Aufsatzes von Romano Guardini. Die Schildgenossen. 1. Jg., H. 4, S. 113-115.

KINDT, Werner, 1963. Grundschriften der Deutschen Jugendbewegung. Düsseldorf - Köln: Diederichs.

KLUG, Ignaz, 1920. Lebensbeherrschung und Lebensdienst. 2. Auflage. Paderborn: Schöningh.

KOCH, Otto, 1913. Die Alkoholfrage. Heidhausen a. d. Ruhr: St. Kamillushaus.

LIEDL, Maria, 1920. Von Ziel und Weg. Die Schildgenossen. 1. Jg., H. 1, S. 13-16.

MAHLICH, Georg, 1921. Was ist Führertum? Die Schildgenossen. 1. Jg., H. 5, S. 145-146.

PLATZ, Hermann, 1920. Anmerkungen zur Burgtagung. Die Schildgenossen. 1. Jg., H. 1, S. 30-32.

POPERT, Hermann, 1910. Helmut Harringa. Eine Geschichte aus unsrer Zeit. Dresden: Päßler. 
PONSCHAB, Karl, 1922. Was heißt Führer sein? Quickborn. 10. Jg., H. 1, S. 191-192. ROCHOLL, Norbert, 1922. Wandervogel und Quickborn. Quickborn. 10. Jg., H. 2, S. 34-37. SPÖRL, Johannes, 1923. Unser Dienst am heiligen Herrn Jesus Christ. Quickborn. 10. Jg., H. 11/12, S. 258-260.

STEIDLE, Robert, 1920. Die innere Tagung. Die Schildgenossen, 1. Jg., H. 1, S. 17-25. STREHLER, Bernhard, 1919/20. Kurzrezension zur Zeitschrift ,Das Heilige Feuer ${ }^{6}$ in der Rubrik ,Büchertafel'. Quickborn. 7. Jg., H. 7, S. 114. THILL, Anton, 1922. Der Urquell unserer Kraft. Quickborn. 10. Jg., H. 1, S. 16-17. WAHRECK (Pseudonym?), 1918/19. In schwerer Zeit. Quickborn. 6. Jg., H. 10, S. 148. WINKLER-HERMADEN, Viktor, 1927. Psychologie des Jugendführers. Jena: Fischer.

\section{Literatur}

FIEDLER, Gudrun, 1989. Jugend im Krieg. Bürgerliche Jugendbewegung, Erster Weltkrieg und sozialer Wandel 1914-1923. Köln: Verlag Wissenschaft und Politik. ISBN 3-8046-8730$\mathrm{X}$.

FUCHS, Ottmar, 2005. Die Pastoral im Horizont der ,unverbrauchbaren Transzendenz Gottes‘ (Karl Rahner). Theologische Quartalsschrift. 185. Jg., H. 1, S. 268-285. ISSN 03421430.

GERBER, Stefan, 2012. Pragmatismus und Kulturkritik. Die politische Kommunikation des deutschen Katholizismus in der Anfangsphase der Weimarer Republik. Historische Zeitschrift. Bd. 294, S. 361-390. ISSN 0018-2613.

GRAF, Rüdiger, 2008. Die Zukunft der Weimarer Republik. Krisen und Zukunftsaneignungen in Deutschland 1918-1933. München: De Gruyter. ISBN 978-3-486-70726-7.

GROPPE, Carola, 2008. Erziehung, Sozialisation und Selbstsozialisation als epochale Leitbegriffe und Deutungsmuster. In: MAROTZKI, Winfried u. WIGGER, Lothar, Hrsg. Erziehungsdiskurse. Bad Heilbrunn: Klinkhardt, S. 75-93. ISBN 978-3-7815-1583-3.

HERRMANN, Ulrich, 2006. Wandervogel und Jugendbewegung im geistes- und kulturgeschichtlichen Kontext vor dem Ersten Weltkrieg. In: HERRMANN, Ulrich, Hrsg. ,Mit uns zieht die neue Zeit... : Der Wandervogel in der deutschen Jugendbewegung. Weinheim: Juventa. ISBN 978-3-7799-1133-3.

JANKE, Pia, 2010. Politische Massenfestspiele in Österreich zwischen 1918 und 1938. Wien: Böhlau. ISBN 978-3-205-78524-8.

KASPER, Tomáš, 2016. Die Deutsche Jugendbewegung in der Tschechoslowakei 19181933. In: BRENNER, Christine, BRAUN, Karl u. KASPER, Tomáš, Hrsg. Jugend in der Tschechoslowakei. Konzepte und Lebenswelten (1918-1989). München: Vandenhoeck \& Ruprecht, S. 25-59. ISBN 978-3-525-373118.

KLÖNNE, Arno, 1981. Die ,Deutsche Jugendfront‘. Eine bündische Gruppe im antifaschistischen Exil. Jahrbuch des Archivs der Deutschen Jugendbewegung. Bd. 13, S. 51-64. ISSN 0587-5277.

KLÖNNE, Irmgard, 1990. ,Ich spring' in diesem Ringe: Mädchen und Frauen in der deutschen Jugendbewegung. Pfaffenweiler: Centaurus-Verlag. ISBN 3-89085-264-5. 
LANGHANS, Daniel, 1990. Der Reichsbund der deutschen katholischen Jugend in der Tschechoslowakei 1918-1938. Bonn: Kulturstiftung der deutschen Vertriebenen. ISBN 388557-084-X.

MAIER, Alexander, 2017. Die ,Entfehlerung‘ der Moderne. Katholische Selbstbildung im Quickborn und bei Romano Guardini. In: CONRAD, Anne u. MAIER, Alexander, Hrsg. Erziehung als ,Entfehlerung: Weltanschauung, Bildung und Geschlecht in der Neuzeit. Bad Heilbrunn: Klinkhardt, S. 169-184. ISBN 978-3-7815-2139-1.

MAIER, Alexander, 2018a. Abstinenz als ,soziale Arbeit'. Katholische Selbstbildung und gesellschaftlicher Fortschritt im ,Quickborn' 1909-1919. In: FRANKE-MEYER, Diana u. KUHLMANN, Carola, Hrsg. Soziale Bewegungen und Soziale Arbeit. Von der Kindergartenbewegung zur Homosexuellenbewegung. Wiesbaden: Springer VS, S. 77-90. ISBN 978-3-658-18590-9.

MAIER, Alexander, 2018b. Christus erleben - Aspekte einer Pädagogik der Gleichzeitigkeit in der Quickborn-Bewegung und bei Romano Guardini. In: MAIER, Alexander, CONRAD, Anne, WEBER, Jean-Marie u. VOSS, Peter, Hrsg. Lernen zwischen Zeit und Ewigkeit. Pädagogische Praxis und Transzendenz. Bad Heilbrunn: Klinkhardt, S. 78-90. ISBN 978-37815-2255-8.

MAKROPOULOS, Michael, 1994. Haltlose Souveränität. Benjamin, Schmitt und die Klassische Moderne in Deutschland. In: GANGL, Manfred u. RAULET, Gérard, Hrsg. Intellektuellendiskurse in der Weimarer Republik. Zur politischen Kultur einer Gemengelage. Frankfurt am Main - New York: Campus-Verlag, S. 197-211. ISBN 3-593-35003-3.

MARMETSCHKE, Katja, 2006. ,Nicht mehr Jugendbewegung, sondern Kulturbewegung! . Die Zeitschrift Die Schildgenossen in der Weimarer Republik. In: GRUNEWALD, Michel u. PUSCHNER, Uwe, Hrsg. Das katholische Intellektuellenmilieu in Deutschland, seine Presse und seine Netzwerke 1871-1963. Bern: Lang, S. 280-317. ISBN 3-03910-857-3.

MERGEL, Thomas, 2005. Führer, Volksgemeinschaft und Maschine. Politische Erwartungsstrukturen in der Weimarer Republik und dem Nationalsozialismus 1918-1936. In: HARTWIG, Wolfgang, Hrsg. Politische Kulturgeschichte der Zwischenkriegszeit 19181939. Göttingen: Vandenhoeck \& Ruprecht, S. 91-127. ISBN 978-3525364215.

NIEMEYER, Christian, 2013. Die dunklen Seiten der Jugendbewegung. Vom Wandervogel zur Hitlerjugend. Tübingen: Francke Attempto. ISBN 978-3-7720-8488-1.

OELS, David und SCHNEIDER, Ute, Hrsg, 2015. ,Der ganze Verlag ist einfach eine Bonbonniere: Ullstein in der ersten Hälfte des 20. Jahrhunderts. Berlin: De Gruyter. ISBN 978-3-11-033708-2.

REULECKE, Jürgen, 2001. ,Ich möchte einer werden so wie die...' - Männerbünde im 20. Jahrhundert. Frankfurt am Main: Campus-Verlag. ISBN 3-593-36727-0.

REULECKE, Jürgen, 2011. Eine junge Generation im Schützengraben. ,Der Wanderer zwischen beiden Welten' von Walter Flex (1916/17). In: LAAK, Dirk van, Hrsg. Literatur, die Geschichte schrieb. Göttingen: Vandenhoeck \& Ruprecht, S. 151-164. ISBN 978-3-52530015-2. 
REULECKE, Jürgen, 2013. Der jugendbewegte Neuaufbruch nach 1918. Die bündische Jugend und ihre Formen der Vergemeinschaftung. In: GROSSMANN, G. Ulrich, Hrsg. Aufbruch der Jugend. Deutsche Jugendbewegung zwischen Selbstbestimmung und Verführung. Ausstellungskatalog des Germanischen Nationalmuseums. Nürnberg: Verlag des Germanischen Nationalmuseums, S. 52-57. ISBN 978-3-936688-77-1.

RICHTER, Reinhard, 2000. Nationales Denken im Katholizismus der Weimarer Republik. Münster: Lit. ISBN 3-8258-4991-0.

RUDDIES, Hartmut, 1994, Florierende Versatzstücke und ideologische Austauscheffekte. Theologische Antworten auf die Ambivalenz der Moderne. In: GANGL, Manfred u. RAULET, Gérard, Hrsg. Intellektuellendiskurse in der Weimarer Republik. Zur politischen Kultur einer Gemengelage. Frankfurt am Main - New York: Campus-Verlag, S. 19-35. ISBN 3-593-35003-3.

RUSTER, Thomas, 1994. Die verlorene Nützlichkeit der Religion. Katholizismus und Moderne in der Weimarer Republik. Paderborn: Schöningh. ISBN 3-506-77381-X.

SCHRÖDER, Peter, 1996. Die Leitbegriffe der deutschen Jugendbewegung in der Weimarer Republik. Eine ideengeschichtliche Studie. Münster: Lit. ISBN 3-8258-2827-1.

TAYLOR, Charles, 2013. Die Formen des Religiösen in der Gegenwart. Frankfurt am Main: Suhrkamp. ISBN 978-3-518-29168-9.

WEICHLEIN, Siegfried, 2015. Zwischenkriegszeit bis 1933. In: DINZELBACHER, Peter, Hrsg. Handbuch der Religionsgeschichte im deutschsprachigen Raum, Bd. 6/1: 20. Jahrhundert - Epochen und Themen. Paderborn: Schöningh, S. 61-112. ISBN 978-3506-72025-2.

WEISS, Otto, 2014. Kulturkatholizismus. Katholiken auf dem Weg in die Deutsche Kultur 1900-1933. Regensburg: Pustet. ISBN 978-3-7917-2615-1. 